\title{
An epigenetic machine
}

\author{
Review: Marcello Barbieri, \\ The Organic Codes: An Introduction to Semantic Biology. \\ Cambridge: Cambridge University Press, 2003.
}

\section{Anton Markoš ${ }^{1}$, Eduard Gajdoš, László Hajnal, Fatima Cvrčková}

\begin{abstract}
Attempts to grasp the essence of the living can be grounded in different areas of human knowledge. If we set aside theological or mythical explanations, we are left with two basic approaches. Biology as a natural science prefers assuming pre-existent and well defined entities, subjects of immutable laws and therefore discernible, describable and computable. In contrast, "humanities" (semiotics, hermeneutics, philosophy and the like) stress the historical and contextual aspect of the "lifeworld", i.e. namely those properties that cannot be covered by constructs of physics. Both approaches are mutually incompatible and the trench dividing them seems to be insurmountable. Perhaps the best difference between them can be perceived in their approach toward concepts like information. Whereas in natural sciences it represents a computable and measurable entity, in humanities it is an entity that, in spite of its immaterial and unquantifiable nature, exerts its influence upon the world.

It is therefore both surprising and encouraging when a scientist takes a term used by the humanities and makes a serious effort to incorporate it into the standard toolbox of experimental biology. The term is meaning, and the author is Marcello Barbieri (Barbieri 2003). The principal claim of his book is that contemporary biology fails to understand life properly, because it is focused only on two principal aspects out of three: energy and information. The third aspect - meaning - remains totally neglected. It is meaning through which memory, frozen patterns (e.g. the genetic code), and conventions come to existence in living beings, in contrast to blind causal relations reigning in the realm of the inorganic. Ordinary chemical reactions, for example, will proceed repeatedly and predictably according to their energy charge and external conditions (e.g., temperature). No such causality
\end{abstract}

1 Author's address: Anton Markoš, Department of Philosophy and History of Science, Faculty of Sciences, Charles University Prague, Viničná 7, 12844 Praha 2, Czechia; e-mail: markos@natur.cuni.cz. 
is obvious in case of processes like protein synthesis. Without doubt, energy to drive the process must be available, but digital "information" in the form of mRNA is also required. The synthesis of a polypeptide would not, however, proceed without a third factor, connecting the realm of the nucleic acids with that of proteins: the system of tRNAs (and code-bearing enzymes aminoacyl-tRNA-synthetases). Thanks to this interface, determining how a specific non-random polypeptide chain is synthesized, proteosynthesis gains its meaning. If we dare to incorporate meaning into biology, states the author, we shall witness reformulation of both great biological narratives: evolution and ontogeny. "Evolution by natural selection" would turn into "Evolution by natural selection and natural conventions". Similarly "Ontogeny as an execution of a program" will be reformulated as "Ontogeny as epigenetic reconstruction from (genetic) projections". What follows is partly a review of Barbieri's book, partly discussion and comments on the main issues of his work. We investigate both the firmness of the proposed footbridge across the gap and possible ways of further reinforcing its construction.

\section{Information, meaning, code: a language analogy}

"Meaning is an object, which is related to another object via a code", says Barbieri (p. 5), and to illustrate the statement, he takes a language analogy:

Mental objects (meanings) are related to objects of the world by the language code, i.e., words. We can easily measure the amount of information ${ }^{2}$ in a world, say "ape" - which equals the number of bits necessary to pick the letters from a given alphabet and align them in a given order. Information, however, has nothing to do with the meaning of that world in different languages (e.g. "ape" in English and Italian). In contrast, the world for male family progenitor has diverged substantially in Indo-European languages: words pater, father, père, etc. obviously contain different amounts of information, in spite of their identical meaning. (p. 94)

This analogy, however, brings more questions than explanations: (1) Does it suggest that languages were devoid of information before the invention of alphabetic (i.e. digital) script? (2) Is information simply a matter of spelling? Can we change the "information content" of words simply by changing orthography? If so, what is such "information" good for? (3) Are words "mere labels" for things out there in the world, having no meaning by themselves? Sure, the string APE as such has no meaning, but a string of 3 letters is not a word. Only after we state that this string represents a word (not a thing!) in, say, English, a plethora of possible dictionary meanings will pop out immediately, and the context will decide which one we take. Words do

2 In the technical, Shannonian sense. 
have meanings; strings of letters may or may not. Such strings, however, can evoke an interpretative effort, which may create meaning, but we believe that meaning is not there. Strings like padre, père, father, Vater will gain their meaning only in an environment where there is somebody (a sentient being) speaking the respective language(s) and, moreover, able to read. All such questions and parables can be brought back to a biological context.

The whole analogy, however, can be turned upside down. One can argue that language is not only a code; language, not the alphabet, makes words; and language defines, makes, creates, not simply connects objects, mental or "real". As stated by J. Lotman (e.g., 2001), should the addressee and the receiver share identical information or text, it is necessary that they share authentic coding and decoding devices. What is possible theoretically (and in technology), will never be attained in "live" communication systems. Culture, language, texts, consciousness (and living beings, we add, to complement Lotman's list) work in two directions: they create a unified semiotic situation to allow exchange of messages and, at the same time a dis-unified situation creating new texts, new information. Communication or reading thus means breaking of symmetries: the original entanglement of many possible meanings will "collapse" into a single interpretation; the interpretation attained will, in its turn, become new superposition of new statements, etc. The body of a culture, a language (and a living being, we add) represent thus structures which can act as their own inputs. In this way the structure is able of self-transformation.

In coding, similar superpositions are forbidden: codes cannot acquire new information. Only thanks to this property it is possible to quantify coded information. An example of an unequivocal code is a transcription between two sets of signs, i.e. the English alphabet and the Morse "alphabet" ("c" is transformed, by convention, as "_-_." ). ${ }^{3}$ Note that only whole tables of codes exist, in which all conventions are contained at the same time. For codes, history is forbidden, says Lotman - codes cannot evolve, they can be only changed as a whole by a single synchronous decision act. We are not interested in how the code came into existence. Since it does not change in the time interval of our interest, rules of transcription can be programmed into machines (or to ribosome's in the cell). No subject is necessary to do the job; the code is independent of the context. ${ }^{4} \mathrm{~J}$. Monod (1970) acknowledged the existence of codes by the term gratuity, which meant that some functions will

3 Equivocal or degenerate codes do also exist. For example, in Czech "c" is also transcribed as "-_-.", but backward translation allows both "c" and "č". Similar situation in the genetic code.

4 Even if sometimes it can be dependent on its position in the string. The genetic codon AUG codes for the amino acid methionine. In bacteria, however, AUG at the beginning of the string will code for $\mathrm{N}$-formyl methionine. 
be mediated "for nothing", simply thanks to existing conventions, rules, not deducible from natural "laws".

Thirty years after Monod, Barbieri tries to broaden gratuity also to natural languages by stating that written and spoken languages are also nothing but a code connecting objects of the world with mental objects. Of course we can find areas, in language, where unequivocality is a goal and codes prevail, like in military. Outside such special areas, however, grammar is overwhelmed by semantical, semiotical, or hermeneutical levels: contexts, experience, situation enter the game, which is accessible to a sentient subject, not a machine. Similar levels can be distinguished also in proteosynthesis, where the genetic code represents one important level of control out of many, not all of them hardwired (see Markoš 2002).

Barbieri, however, suggests hardwired codes not as a derived situation but as the very basis of meaning. This is a very courageous reduction: Even the most elementary usage of the word meaning ${ }^{5}$ excludes its usage in a sense of "context-independent decoding rule". More sophisticated usage — as, e.g., intention, purpose, spirit of the told (or written), interpretation, signification - points clearly towards a conclusion that meaning cannot be subject of any coding table or context-independent rule. Only when we accept this, can we speak - in a natural language — of semantics.

Barbieri in contrary intends to introduce meaning in a technical sense, similarly as Shannon did for information. He explicitly states:

The term codes, or conventions, normally indicates the rules which are adopted by a human community, but it has also a wider meaning. A code can be defined as a set of rules that establish a correspondence between two independent worlds. The Morse code, for example, connects certain combinations of signs with letters of the alphabet. The highway code is a liaison between illustrated signals and driving behaviors. A language makes words stand for real objects of the physical world. (p. 89, emphasis by us)

This sentence brings us to a very strange world, where "real objects" stick "out there" and we simply attach our linguistic labels (i.e. codes) to them. No semantics and no semiotic process are allowed in this world, where meaning is indistinguishable from code! This may hold only in some variety of perfect languages, be it artificial languages, computer language, or mathematical calculus (see, e.g., Eco 1997).

The quotation above, moreover, continues as follows: "The extraordinary thing about codes is that a new physical quantity appears in them, since they require not only energy and information but also meaning“ (p. 94). In what sense codes are "physical quantities"?" $\mathrm{We}$ argue that meaning is a

As in "What should this all mean?"

An effort to make virtual entities real and thus "justify" their usage in science is apparent from sentences like "codes [...] must have had a specific mechanism" 
relationship, which must be negotiated in every particular situation. There may be a finite table of codes, but never of meanings (see, e.g, Heelan 1998: 279,288 ). Meaning is understanding, not a table of codes.

\section{The logic of embryonic development}

In the 1940s, C. H. Waddington (1975) introduced the concept of epigenetics to give a name to causal interactions between genes and their products that lead to the accomplishment of the phenotype. Today, epigenetics serves practically as a synonym for ontogeny; its meaning is occasionally broadened also to heritable changes in gene function, to innate operations taking place in the brain, practically to any biological patterning which does not involve changing DNA sequences (examples of such usage see, e.g., Lodish et al. 1995: 1286; Russo et al. 1996: 1; Wilson 1998: 193, respectively). What all such perspectives have in common is that they see development as a process being canalized along some preexisting trajectory (program); the trajectory may branch, i.e. contain alternative subroutines called forth by the environment. The living being remains fully passive, without any say in the ongoing ontogeny; no meaning is necessary to understand the process.

Barbieri is, rightly, not happy with this neat preformist world where novelty can arise only by random mutation. Instead he presents a view of epigenetics as a convergent process ${ }^{7}$ of reconstructing structures from projections (i.e. from incomplete information, p. 3). ${ }^{8}$ Information contained in the zygote or spore is somehow insufficient, unsatisfactory, and new information must be generated to build an adult organism. "The reconstruction of structures from incomplete information is therefore a model that could make us understand how it is possible for a system to obtain a convergent increase of complexity" (p. 70). Notice: information must be created, a great leap forward from the traditional views hold by informatics. Author provides an original model (MCM, see below) how such a process might take place.

(p. 2); "mechanism of natural conventions" (p. 2); "organic codes are not metaphorical but real" (p. 3); "organic code requires molecules" (p. 3), etc. Also the physical terminology is not new - see Driesch's definition of entelechy as a "physical quantity" (Driesch 1905).

7 Terminological note: ontogeny is understood as convergent, whereas evolution as divergent, increase of complexity.

8 "As anyone can see, this is a mathematical version of the problem that we face in embryonic development. The fertilized egg contains far less information than the adult organism (whatever criterion is used to measure information in biological systems), and embryonic development can be described therefore as a process that is reconstructing a structure from incomplete information" (p. 68).

9 It is a priori presupposed that the adult form is the very goal of ontogeny. 


\section{The cell}

To understand further reasoning, we make a short detour to the cellular level. The cell is depicted (pp. 34-35) as, (a) something which builds itself like a crystal (obviously a view inspired by self-assembly of viral particles or ribosomes); apparently the absence of beginning poses no problem; (b) a "machine capable of self-replication" as in von Neumann automata; (c) an autopoietic system.

Obviously, Barbieri considers these three descriptions as practically synonymous. Indeed, he apparently treats the whole ontogenesis of an ape or a bee as a mere assembly of a body from organs, organs from tissues, tissues from cells etc. (pp. 95-96). We believe that this extrapolation, well in the line with the famous "an elephant is just $E$. coli writ large", deserves a word of caution, instead of being treated as a simple and indisputable fact. If not, we will remained marooned in contradictions like "a mechanical model of epigenesis". 10

\section{Ontogeny as program execution}

As already mentioned, Barbieri is criticizing the naive self-satisfaction exerted by some molecular biologists that "we know by now" that development is simply an execution of a program inscribed in genes. ${ }^{11}$ Barbieri takes Maynard Smith as an authority to corroborate this opinion, and continues: "embryonic development is a process that increases the complexity of a living system, but we do not know how to build machines that increase their own complexity, and we cannot therefore understand the logic of development" (p. 67-68). It can be argued whether the logic of such a process can be understood only through modeling it as a machine, i.e. by deterministic rules. The question of "how does a system manage to increase its own complexity in a convergent way?" was more or less satisfactorily answered by mathematical models for systems, which are able to increase their complexity, like whirl-pools, tornadoes, or even biosphere (see, e.g., Kauffman 2000; Prigogine 1980). It is simply not true that "there cannot be a convergent increase of complexity without memory" (p. 86), or better, the

10 "We need to understand how does a system manage to become more complex, otherwise the word "epigenesis" becomes a mere label that is conveniently used only to cover up our ignorance, just as "vis vitalis" in the past. We need, in other words, a mechanical model of epigenesis in order to understand it. Luckily today we do have such a model [the one presented], and we can at least try to apply it to the cell" (p. 212).

11 See, for example, Davidson (2001: 7): "It was possible to deduce that genomic regulatory architecture constitutes the structural, genetic basis for the morphological features of animals 30 years ago; now we know it for a certainty". 
memory may sometimes reside in the very structure of the "body" of the system.

Back to the logic of embryonic development. "The real key to embryonic development is the logic of systems which are capable of increasing their complexity in a convergent way, and in order to understand this we need if not a machine, at least a model that is functioning according to that logic" ( $p$. 68). The model offered by Barbieri is rooted in his rich experience in image analysis and reconstruction of the shape of three-dimensional objects from two-dimensional projections. Such a task can be solved relatively easily if there are enough data (projections) available, so that they "contain (in a compressed form) all the information that was present in the original structure" (p. 69). ${ }^{12}$ However, surprisingly good results can be obtained even with substantially less data, if we employ special iterative algorithms such as the Memory Reconstruction Method (MRM), developed by the author. The model exploits memory matrices as very suitable to describe the logic of embryonic development.

\section{Increase of complexity}

If we have a model for the reconstruction of structures from incomplete information, it will help us understand how it is possible for a system to obtain a convergent increase of complexity. However, what does it mean if we say that the egg contains less information than an adult does, what is meant by the convergent increase of complexity? Barbieri admits that "there is no satisfactory definition of complexity. However we do not need to provide a precise definition of complexity in order to build a model", because "we can start from a different formulation of the problem, and say that embryonic development is a reconstruction from incomplete information" ( $p$. 196). The difference between complexity and information is fuzzy, but we leave these details aside for the moment. For a closer approach to the convergent increase of complexity, let us discuss two examples.

The first comes from R. Dawkins' famous definition: "a complex thing is something whose constituent parts are arranged in a way that is unlikely to have arisen by chance alone" (Dawkins 1987). If we jumble parts of an airliner at random, the likelihood that a working Boeing 747 will come out spontaneously is vanishingly small. Only one or very few contraptions out of zillions would actually fly. The arrangement of the parts that flies is meaningful and the other arrangements are meaningless regardless the fact, whether the right arrangement of the parts was specified in advance or not.

Certainly, the documentation does not contain the "complete" information for the construction of an aircraft. No project will bother with providing

12 Again we face the question what is meant by "all the information". 
information concerning, e.g., how to screw bolts or use a hammer, not speaking about iron-ore mining instructions, theory of wheel, and Newtonian mechanics... No project is accomplished in 3 dimensions and in 1:1 ratio, to contain the complete information. It has to be detailed enough to be understandable for the comprehensive reader or even for a robot equipped with necessary instructions. Let us now imagine 747 documentation with some pages and drawings missing. It may even appear that only as little as $10 \%$ of the files is really crucial for the construction of a functional plane. Plans for kitchens, seats, bathrooms, seatbelts etc. could be supplied by anyone who understands what kitchens, seats, bathrooms, seatbelts etc. are good for, i.e. what they mean. Somebody who understands aeronautical engineering may even do it without all those detailed mechanical drawings; he even might be able to assemble the parts in a better way than what was intended in the (now lost) documentation. Any (re)construction requires a big deal of experience and/or constructive imagination.

Barbieri's book will serve as our second example. Certainly, the book does not contain all the information on semantic biology, organic codes, ribotype, phenotype etc.; all these items are much more complicated than the book itself. Is it a deadlock forbidding our understanding of the book? We hope not, we have even methods, how to reconstruct that missing information, how to complete the picture, even how to understand better the intentions of the author. We have to read the book carefully again and again, to interpret it and to remember what was read and interpreted, and confront it with our own experience and knowledge.

The message of both our examples is as follows: any reconstruction from a projection requires a comprehensive reader who understands what is to be reconstructed. Otherwise that blank could be filled only by pre-defined rigid structures given in advance, but these are surely not the concern of semantic biology.

\section{Increase of information}

An increase of information in the Shannonian sense within a system is inconceivable. Information can increase only if (1) a wired comprehensive model exists for the reconstruction of structures (i.e. the model contains additional information), or (2) an understanding, informed reader can build a whole structure from incomplete source (see above the 747 example). In its core, the MRM is a mathematical compression/reconstruction model, which accepts that "there cannot be a convergent increase of complexity without memory" (p. 90). The problem of the MRM model is not in the model itself, as it is used for reconstruction of 3-dimensional structures from a limited set of 2-dimensional projections, but in its use as a biological metaphor. Therefore, it may be even an advantage that it is vague as concerns the nature 
of the "organic memory", which plays a crucial role in the reconstruction process and "generating information."

The task of compression/reconstruction consists of two components, an encoding algorithm that takes a message and generates a "compressed" representation, and a decoding algorithm that reconstructs the original message or some approximation from the compressed representation. These two components are intricately tied together since they both have to understand the shared compressed representation. The methods can be divided into two types: Lossless algorithms (typically used for texts) reconstruct the original message from the compressed message exactly, whereas lossy algorithms (used for images and sounds) recover with somewhat lower resolution. Methods can also be classified as either static or dynamic. In a static mode, the mapping from messages to codewords has been fixed before the transmission begins, so that a given message is represented by the same codeword every time it appears in the message ensemble (Huffman coding). In a dynamic mapping, the set of codewords changes over time. An algorithm may also be a hybrid of both static and dynamic regimes. The MRM, in this respect, can be characterized as a static lossy reconstruction model.

\section{Reconstruction}

Barbieri takes morphogenesis as a reconstruction (of an adult) from an $\mathrm{n}$ dimensional "projection" (DNA sequence) or from a bunch of projections (images) represented by the zygote. The first problem of this approach lies in the far from obvious translation (rather than coding) of a bodily structure into a digital string of the genetic text. In the model, it is us who decide (1) what properties of the object should be "scanned" and (2) what the accuracy of digitalization (size of "pixels") should be. ${ }^{13}$ Who or what is the decisionmaker in zygote - body transformations?

The process of reconstruction, in the model, resides in an interplay between the state present at the beginning, and a "memory matrix", which is empty at the beginning. (Note that there is a beginning when all parameters of a living being are reset to "time zero".) The model works as follows:

The initial memory matrix is a tabula rasa, a white page that is gradually filled during the reconstruction process, while the reconstructed picture starts with a uniform image and becomes progressively differentiated in the course of time. A reconstruction with a MRM method, in other words, is a set of two distinct reconstructions that are performed in parallel. The point is that this double reconstruction is necessary for reasons which are absolutely general. (p. 90)

13 Note that it cannot be refined ad infinitum because of the uncertainty principle. 
The whole model is reminiscent of bootstrapping of a computer upon startup. We can view the relatively small and simple system files as "projections" from which the complex and in some aspects almost lifelike "image" of the running operation system is produced by action of a "codemaker" (hardware and BIOS) and a "memory matrix" (RAM). Completeness or incompletness of system files is just a matter of complexity of the hardware and BIOS and/or RAM size.

We have thus two independent worlds: that of an image or projection and that of organic memory. They intensively communicate in both ways, which leads to filling the memory and, at the same time, elaborating the picture. As the nature of both worlds is different, the flow of communication between them can be accomplished only thanks to the existence of suitable conventions called organic codes. Embryonic development is possible, states Barbieri, only if organic codes and organic memories are in charge. If "resetting" to time zero really takes place in a zygote, then epigenesis might indeed work according to the model.

The principal question, however, is why do we need to introduce meaning into such a system? The whole model is indeed a variation of the computer metaphor, with a concealed presence of a creator of the computer in the background - only in his/her head there is something like meaning.

\section{The third space}

Even if the book is not really about meaning as the author suggests, but rather about decoding, it still touches the enigma how two worlds - that of "immaterial" digital symbols, epitomized as genotype and that of "material" bodies, shapes, and patterns (phenotypes) - can become related. According to Barbieri, the interface is codes. However, here comes to the focus the question of their origin and of the codemaker (and of course also the decoding entity).

A paradigmatic (if by no means single) example of coding in living system is presented by a set of tRNAs, representing the ribotype, mediating between the genotype and phenotype, although many elaborated coding systems exist at different levels of organization. The effects that external [we add: also internal] signals have on cells, in conclusion, do not depend on the energy and the information that they carry, but only on the meanings that cells give them with rules that can be called signal transduction codes" ( $\mathrm{p}$. 106). Yes, most of "reactions" 14 going on in the cell are important not metabolically, but semantically. But if it is the cell that gives a meaning to the signals, does it mean that these signals - in contrast to the genetic code -

14 We put quotation marks because many cellular signaling processes represent rather molecular recognition than standard chemical reactions on covalent bonds. 
are not universal? Not only in the sense of frozen accidents but also as frozen conventions? Barbieri may be disposed towards such an explanation: "This implies that there is in cells an equivalent of the contextual information that plays such a relevant role in language" (p. 107).

We fully agree, but as we have stated above, language is not only a coding system. Everything is prepared here for semantic biology, at a price: biology will not remain in the realm of (fully?) experimental science. Barbieri is not willing to take this last step. He will protest against "frozen accidents", he will even suggest that the barrier between nature and culture should be brought down. He does not say it explicitly, but we can interpret his words as follows: if we have a "countless number" of "codes" in living beings, then living beings can be taken as an analogy of culture. One of us (Markoš 2002) has developed a similar analogy, arguing that a species can be understood as an analogy of culture, with its internal language and convention how to interpret its own living according to internal or external cues. What we are uneasy with, is the antinomy code-language. Codes cannot be used to tell a story. With codes, living beings remain safely in the realm of science, with language they cross the barrier towards "humanities". We can, of course, try to bring natural language to science's side of the barrier. Such a move would, however, change substantially science as we know it.

\section{Semantic biology}

Can we constitute a semantic biology? Barbieri gives a positive answer, but the more we read, the more we suspect that what is being discussed is not semantic but syntactic biology, in other words, a kind of grammar and (complete sets of) rules of a biological "language" (in the sense of computer language). But how can we know at what moment the code became "complete"? Why it must not be modified? Is not the only reason for such a claim that it is necessary to stick to timeless "laws" in order to stay in the realm of science? We remind again the Morse code, created indeed as a complete set. However, the fullness of a statement's meaning lies not in its internal grammatical or logical structure but in its ability to illuminate the totality of fore-understandings which are the grounds of its intelligibility. In this context, we should refer to another contemporary book, which also attempts to constitute a general biology: Investigations by S. Kauffman (2000). But Kauffman acknowledges internal activity in his autonomous agents, conventions in his models are truly generated and changing in evolution - they are product and subject of history, not of a list of given immutable rules!

We agree with the general conclusion that "every cell must have (1) organic structures, (2) organic memories, and (3) organic codes" (p. 212; albeit organic seems to be a mere epitheton ornans). However, we maintain 
that these pre-requisits, although necessary, are not sufficient, and that in order to grasp life, we have to introduce yet another component, very close to what we call habits in our culture. ${ }^{15}$

\section{References}

Barbieri, Marcello 2003. The Organic Codes: An Introduction to Semantic Biology. Cambridge: Cambridge University Press.

Davidson, Eric H. 2001. Genomic Regulatory Systems. Development and Evolution. San Diego: Academic Press.

Dawkins, Richard 1987. The Blind Watchmaker. Harlow: Longman Scientific \& Technical.

Driesch, Hans 1905. Der Vitalismus als Geschichte und als Lehre. Leipzig: J.A. Barth

Eco, Umberto 1997. The Search for the Perfect Language. London: Fontana Press.

Heelan, Patrick A. 1998. The scope of hermeneutics in natural science. Studies in the History and Philosophy of Science 29(2): 273-298.

Kauffman, Stuart A. 2000. Investigations. Oxford: Oxford University Press.

Lodish, Harvey; Baltimore, David; Berk, Arnold; Zipursky, S. Lawrence; Matsudaira, Paul; Darnell, James 1995. Molecular Cell Biology (Scientific American Books). W. H. Freeman.

Lotman, Yuri M. 2001. Universe of the Mind: A Semiotic Theory of Culture. London: I.B. Tauris.

Markoš, Anton 2002. Readers of the Book of life: Conceptualizing Developmental Evolutionary Biology. Oxford: Oxford University Press.

Monod, Jacques 1970. Le hasard and la nécessité. Paris: Seuil

Russo, Vincenzo E. A.; Martienssen, R. A.; Riggs, A. D. (eds.) 1996. Epigenetic Mechanisms of Gene Regulation. Cold Spring Harbor: Cold Spring Harbor Laboratory Press.

Prigogine, Ilya 1980. From Being to Becoming: Time and Complexity in the Physical Sciences. San Francisco: Freeman \& Co.

Waddington, Conrad H. 1975. The Evolution of an Evolutionist. Edinburgh: Edinburgh University Press.

Wilson, Edward O. 1998. Consilience: The Unity of Knowledge. Alfred A. Knopf.

15 Acknowledgements. We thank the Grant Agency of the Czech Republic (Grant 401/02/0636, AM; Grant 195/2002/B-FYZ/CTS, EG) and Ministry of Education of the Czech Republic (Project J13/98:113100003, FC) for financial support. 